\title{
Kv/Mv Imaging
}

National Cancer Institute

\section{Source}

National Cancer Institute. Kv/Mv Imaging. NCI Thesaurus. Code C116493.

Any imaging method that makes use of high-energy radiation in the Kv or Mv range. 